\title{
Tensile fracture behavior of a Zr-based bulk metallic glass subjected to HPT
}

D.V. Gunderov ${ }^{1,2,3, \dagger}$, E.V. Boltynuk ${ }^{3}$, E.V. Ubyivovk ${ }^{3}$, A.A. Churakova ${ }^{1,2,3}$, A.V. Lukyanov ${ }^{1,4}$,

\author{
A.G. Raab ${ }^{1}$, D.A. Khasanova ${ }^{1}$, A.Yu. Churyumov ${ }^{5}$ \\ †dimagun@mail.ru
}

\author{
${ }^{1}$ Ufa State Aviation Technical University, 12 K. Marx str., 450008 Ufa, Russia \\ ${ }^{2}$ Institute of Molecule and Crystal Physics, Ufa Research Center RAS, 151 pr. Oktyabrya, 450075 Ufa, Russia \\ ${ }^{3}$ Saint Petersburg State University, 28 Universitetskiy pr., 198504 Saint Petersburg, Russia \\ ${ }^{4}$ M.N. Mikheev Institute of Metal Physics IMP UB RAS, 18 S. Kovalevskoy str., 620990, Ekaterinburg, Russia \\ ${ }^{5}$ Nantional University of Science and Technology“MISiS”, 4 Leninskiy pr.,119049 Moscow, Russia
}

The microstructure and mechanical properties of the $\mathrm{Zr}_{62} \mathrm{Cu}_{22} \mathrm{Al}_{10} \mathrm{Fe}_{5} \mathrm{Dy}_{1}$ bulk metallic glass (BMG) processed by high pressure torsion (HPT) at temperatures of $20^{\circ}$ and $150^{\circ} \mathrm{C}$ were investigated. Transmission electron microscopy and XRD studies did not reveal any structural transformations. The material in both initial amorphous state and after HPT processing demonstrates brittle fracture under uniaxial tensile testing at temperatures in the range of $20-300^{\circ} \mathrm{C}$. The maximum fracture stress of the material in the initial state is $1410 \mathrm{MPa}$. Fracture stress of the HPT-processed states is lower in comparison with that of the initial state due to the presence of microcracks in the HPT-processed samples. The values of the maximum fracture stress are 250 and $1240 \mathrm{MPa}$ for the samples processed by $\mathrm{HPT}$ at $20^{\circ}$ and $150^{\circ} \mathrm{C}$, respectively. Fracture surfaces of the material in the initial state and after HPT processing have different morphologies due to structural transformations induced by HPT processing. Annealing of the samples in the initial state and after HPT processing results in smaller grain size in the HPTprocessed samples in comparison with the initial samples. However, microindentation reveals that hardness of the material in the initial state after annealing is noticeably higher than hardness of the HPT processed alloy after the same annealing. Thus, it can be outlined that HPT processing affects the microstructure evolution in the material during further annealing.

Keywords: amorphous alloys, severe plastic deformation, structures, mechanical properties.

\section{Особенности разрушения при растяжении объемного аморфного сплава на основе $\mathrm{Zr}$, подвергнутого ИПДК}

Гундеров Д.В. ${ }^{1,2,3,}$, Болтынюк Е.В. ${ }^{3}$, Убыйвовк Е.В. ${ }^{3}$ Чуракова А.А. ${ }^{1,2,3}$, Лукьянов А.В. ${ }^{1,4}$, Рааб А.Г. ${ }^{1}$, Хасанова Д.А. ${ }^{1}$, Чурюмов А.Ю. ${ }^{5}$

${ }^{1}$ Уфимский государственный авиационный технический университет, ул. К. Маркса 12, 450008, Уфа, Россия ${ }^{2}$ Институт физики молекул и кристаллов УНЦ РАН, пр-т Октября 151, 450075, Уфа, Россия

${ }^{3}$ Санкт-Петербургский государственный университет, Университетский пр. 28, 198504, Санкт-Петербург, Россия

${ }^{4}$ Институт физики металлов им. М.Н. Михеева УрО РАН, ул. С. Ковалевской 18, 620990, Екатеринбург, Россия ${ }^{5}$ Национальный исследовательский технологический ун-т “МИСиС”, Ленинский проспект 4, 119049, Москва, Россия

Исследована микроструктура и механические свойства аморфного сплава $\mathrm{Zr}_{62} \mathrm{Cu}_{22} \mathrm{Al}_{10} \mathrm{Fe}_{5} \mathrm{Dy}$, подвергнутого воздействию интенсивной пластической деформации кручением (ИПДК) при температуре $20^{\circ}$ и $150^{\circ} \mathrm{C}$. Методом просвечивающей электронной микроскопии и РСА не удалось выявить каких-либо структурных изменений. Образцы аморфного сплава до и после ИПДК при испытаниях на растяжение при различных температурах, включая $300^{\circ} \mathrm{C}$, разрушаются хрупко. В исходном аморфном состоянии максимальное напряжение разрушения составляет 1410 МПа. Максимальные напряжения разрушения составляют 250 и 1240 МПа для образцов после ИПДК при $20^{\circ}$ и $150^{\circ} \mathrm{C}$, соответственно. Однако вид излома после испытаний на растяжение образцов до и после ИПдК несколько различается, что отражает изменение структуры в результате ИПДК. Размер зерна в сплаве, подвергнутому ИПДК и отжигу заметно меньше, чем сплава, подвергнутого отжигу из исходного состояния, а микротвердость сплава, подвергнутого ИПДК и отжигу заметно ниже, чем Нv отожжённого исходного образца. Таким образом, предварительная ИПДК, видимо, меняет эволюцию структуры при последующем кристаллизационном отжиге.

Ключевые слова: аморфные сплавы, интенсивная пластическая деформация, структуры, механические свойства. 


\section{1. Введение}

Объектом особого внимания в мировой научной литературе являются аморфные материалы $[1,2]$, в том числе так называемые объемные аморфные материалы или объемные металлические стекла (ОМС) [3]. Данные сплавы имеют специфический состав, что позволяет при литье в формы с быстрым охлаждением получить образцы с аморфной структурой диаметром до 10 мм.

Одним из способов воздействия на ОМС и изменения их свойств, может быть интенсивная пластическая деформация (ИПД) $[4,5]$. ИПД является одним из эффективных способов трансформации структуры металлов и сплавов [4-6]. Проведенные многочисленные исследования показали, что ИПД приводит к сильнейшему изменению структурно-фазового состояния материалов [6-8]. Интенсивная пластическая деформация чистых металлов приводит к измельчению зерен до нанометровых размеров. В многокомпонентных материалах в результате ИПД происходят фазовые превращения, образуются пересыщенные твердые растворы, формируются метастабильные фазы, а в некоторых сплавах достигается аморфизация. В частности, при ИПД была обнаружена аморфизация магнитотвердых сплавов системы $\mathrm{Nd}-\mathrm{Fe}-\mathrm{B}$ [9-10]. В серии работ [11 - 16] была исследована аморфизация при ИПД сплавов с эффектами памяти формы системы TiNi, изучена эволюция и структуры и свойств при последующих отжигах. В работах [17-19] было исследовано воздействие ИПД на аморфные быстрозакаленные сплавы $\mathrm{TiNi}, \mathrm{Nd}-\mathrm{Fe}-\mathrm{B}$, рассмотрены обнаруженные процессы нанокристаллизации при ИПД аморфных сплавов. Концепции трансформации структуры при ИПД сплавов в исходном кристаллическом и аморфном состоянии были дополнительно проанализированы в недавних работах [20-24]. Однако вопросы влияния ИПД на тонкую структуру аморфных сплавов, и в частности, аморфных сплавов на основе $\mathrm{Zr}$ далеко не достаточно исследованы.

\section{2. Материалы и методики исследования}

В настоящей работе исходный аморфный сплав $\mathrm{Zr}_{62} \mathrm{Cu}_{22} \mathrm{Al}_{10} \mathrm{Fe}_{5} \mathrm{Dy}_{1}$ (ат.\%) был получен в виде цилиндрических прутков диаметром 5 мм и длиной 50 мм литьем расплава в медный тигель. Для проведения ИПДК пруток был нарезан на диски толщиной 2 мм. Образцы подвергались ИПДК на 5 оборотов наковален (диаметром 10 мм), при скорости вращения 1 об/мин давлении 6 ГПа при комнатной температуре (КТ) и температуре $150^{\circ} \mathrm{C}$. В результате ИПДК были получены объёмные образцы толщиной 0,2-0,3 мм и диаметром 10 мм.

Прецизионно исследование структуры проводилось методом просвечивающей электронной микроскопии с помощью просвечивающего электронного микроскопа (ПЭМ) ЈЕМ-2100. РСА исследования структуры проводились на приборе Bruker Phaser D2 на медном излучении. Поверхности изломов после разрушения изучали на растровом электронном микроскопе (РЭМ) марки JSM-6390 и QUANTA 200.

\section{3. Результаты и обсуждение}

В соответствии с РСА исходные стержни ОМС имели аморфную структуру (рис. 1). После ИПДК и отжига при $370^{\circ} \mathrm{C}$ видимых изменений рентгенограмм не происходит. После отжига образцов ОМС при $500^{\circ} \mathrm{C}$ на рентгенограмме наблюдаются кристаллические пики, что свидетельствует о кристаллизации аморфной фазы.

Светлопольная просвечивающая микроскопия в сканирующем режиме (СПЭМ) исходного ОМС также показывает в основном аморфную структуру (рис. 2a). В ходе исследования структуры аморфной фазы исходного ОМС при больших увеличениях не наблюдается каких-либо особенностей нанокристаллических образований (рис. 2b).

По СПЭМ-изображению (рис. 2а) видно, что в аморфной фазе присутствуют кристаллиты кубической формы размером до 500 нм - частицы оксида диспрозия $\mathrm{Dy}_{2} \mathrm{O}_{3}$. Присутствие данного оксида известно для подобных циркониевых ОМС [25]. Также в аморфной фазе наблюдаются включения отдельных сферических монокристаллов (сферолитов) или конгломератов кристаллитов с округлыми внешними границами, по-видимому, интерметаллидной фазы на основе $\mathrm{Zr}\left(\mathrm{CuZr}_{2}\right)$. Размеры

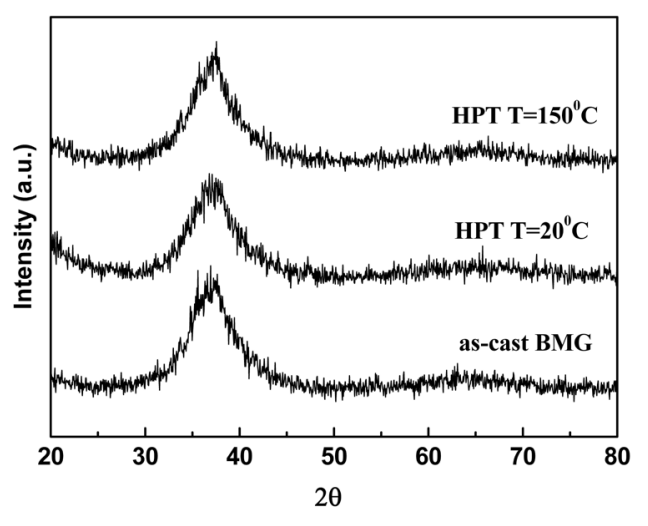

a

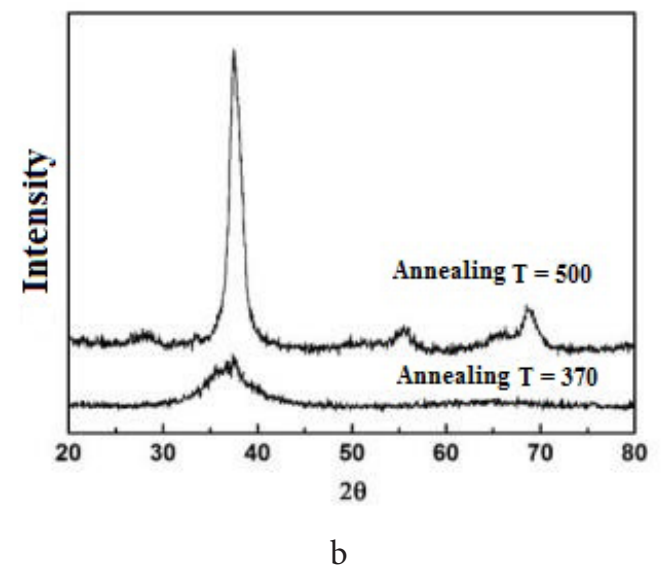

Рис. 1. Картины рентгеновской дифракции для: (а) исходного $\mathrm{Zr}_{62} \mathrm{Cu}_{22} \mathrm{Al}_{10} \mathrm{Fe}_{5} \mathrm{Dy}_{1}$ и образцов, подвергнутых ИПДК при различных температурах; (b) исходный $\mathrm{Zr}_{62} \mathrm{Cu}_{22} \mathrm{Al}_{10} \mathrm{Fe}_{5} \mathrm{Dy}_{1}$ после отжига при температурах $370^{\circ} \mathrm{C}$ и $500^{\circ} \mathrm{C}, 10 \mathrm{Mин}$.

Fig. 1. X-ray diffraction patterns for: (a) initial $\mathrm{Zr}_{62} \mathrm{Cu}_{22} \mathrm{Al}_{10} \mathrm{Fe}_{5} \mathrm{Dy}$ and the samples processed by HPT at different temperatures; (b) initial $\mathrm{Zr}_{62} \mathrm{Cu}_{22} \mathrm{Al}_{10} \mathrm{Fe}_{5} \mathrm{Dy}_{1}$ after annealing at temperatures of $370^{\circ} \mathrm{C}$ and $500^{\circ} \mathrm{C}, 10 \mathrm{~min}$. 
сферолитов и их конгломератов достигают 5-10 мкм. Картины микродифракции, снятые с участка со сферолитами, показывают аморфное гало и рефлексы кристаллической фазы. Однако объёмная доля кристаллов в сплаве невелика (около 5\%), что подтверждается данными РСА. И в целом сплав можно считать аморфным.

Согласно ПЭМ и СПЭМ структура образцов, подвергнутых ИПДК при температурах $20^{\circ} \mathrm{C}$ и $150^{\circ} \mathrm{C}$, остается в целом аморфной (рис. 2d-e). Нанокристаллизации при ИПДК ОМС $\mathrm{Zr}_{62} \mathrm{Cu}_{22} \mathrm{Al}_{10} \mathrm{Fe}_{5} \mathrm{Dy}_{1}$ не обнаружено. A сферолиты, присущие структуре исходного ОМС, в результате ИПДК зачастую разрушаются (дробятся и измельчаются). В тоже время частицы кубической формы оксида диспрозия $\mathrm{Dy}_{2} \mathrm{O}_{3}$ в связи с высокой прочностью, по-видимому, не измельчаются при ИПД и остаются в аморфной фазе (рис 2f).

Механические испытания на растяжение ОМС $\mathrm{Zr}_{62} \mathrm{Cu}_{22} \mathrm{Al}_{10} \mathrm{Fe}_{5} \mathrm{Dy}$ до и после ИПДК проводили на малой разрывной машине УГАТУ, на малых плоских образцах с рабочей базой $0,25 \times 1 \times 3$ мм, со скоростью растяжения $3 \times 10^{-4} \mathrm{c}^{-1}$ при комнатной температуре. Исследования показали, что образцы сплава при растяжении разрушаются хрупко. В исходном аморфном состоянии максимальное напряжение разрушения составляет 1410 МПа. При испытаниях образцов после ИПДК точно определить пределы прочности не удается, видимо, из-за наличия микротрещин в образцах. Максимальные напряжения разрушения составляют 250 и 1240 МПа для образцов после ИПДК при $20^{\circ}$ и $150^{\circ} \mathrm{C}$, соответственно. Значения максимального напряжения разрушения в зависимости от состояния испытаний приведены в таблице 1.
Были проведены РЭМ исследования изломов на ОМС $\mathrm{Zr}_{62} \mathrm{Cu}_{22} \mathrm{Al}_{10} \mathrm{Fe}_{5} \mathrm{Dy}$ в исходном состоянии и в состояниях после ИПДК при температурах $20^{\circ} \mathrm{C}$ (рис. 3,4$)$ после механических испытаний растяжением.

На поверхности разрушения исходного ОМС наблюдается излом, близкий к ямочному с крупными глубокими гребнями отрыва. Среднее расстояние между такими гребнями отрыва составляет около 5 мкм (рис. 3a-b). Область между гребнями отрыва преимущественно не имеет заметного рельефа, скругленное, в форме чаши. В ОМС $\mathrm{Zr}_{62} \mathrm{Cu}_{22} \mathrm{Al}_{10} \mathrm{Fe}_{5} \mathrm{Dy}_{1}$, подвергнутом ИПДК $n=5$ при $T=$ $20^{\circ} \mathrm{C}$ (рис. 4), по первым данным, излом имеет более выраженный «венозный». Расстояние между гребнями отрыва в образце «ИПДК $20^{\circ} \mathrm{C»} \mathrm{составляет} \mathrm{около} 10$ мкм (рис. 4а).

Можно предположить, что это свидетельствует об уменьшении локальной пластической деформации в области разрушения. Однако другое характерное отличие поверхности разрушения $\mathrm{OMC} \mathrm{Zr}_{62} \mathrm{Cu}_{22} \mathrm{Al}_{10} \mathrm{Fe}_{5} \mathrm{Dy}_{1}$ «ИПДК $20^{\circ} \mathrm{C}$, в том, что области между крупными гребнями отрыва имеют внутренний рельеф, т. е. области между гребнями отрыва дополнительно фрагментируются «вторичными» гребнями отрыва (рис. 4b). Капли, которые сформировались в вершинах гребней отрыва в процессе локального нагрева на поверхности разрушения ОМС «ИПДК $20^{\circ} \mathrm{C»,} \mathrm{стали} \mathrm{более} \mathrm{дисперсными.} \mathrm{Можно}$ предположить, что это свидетельствует об увеличении локальной пластической деформации в области разрушения. Таким образом, полученные данные свидетельствуют о некотором сложном изменении процессов разрушения в результате изменения структуры аморфной фазы ОМС после воздействии ИПДК.
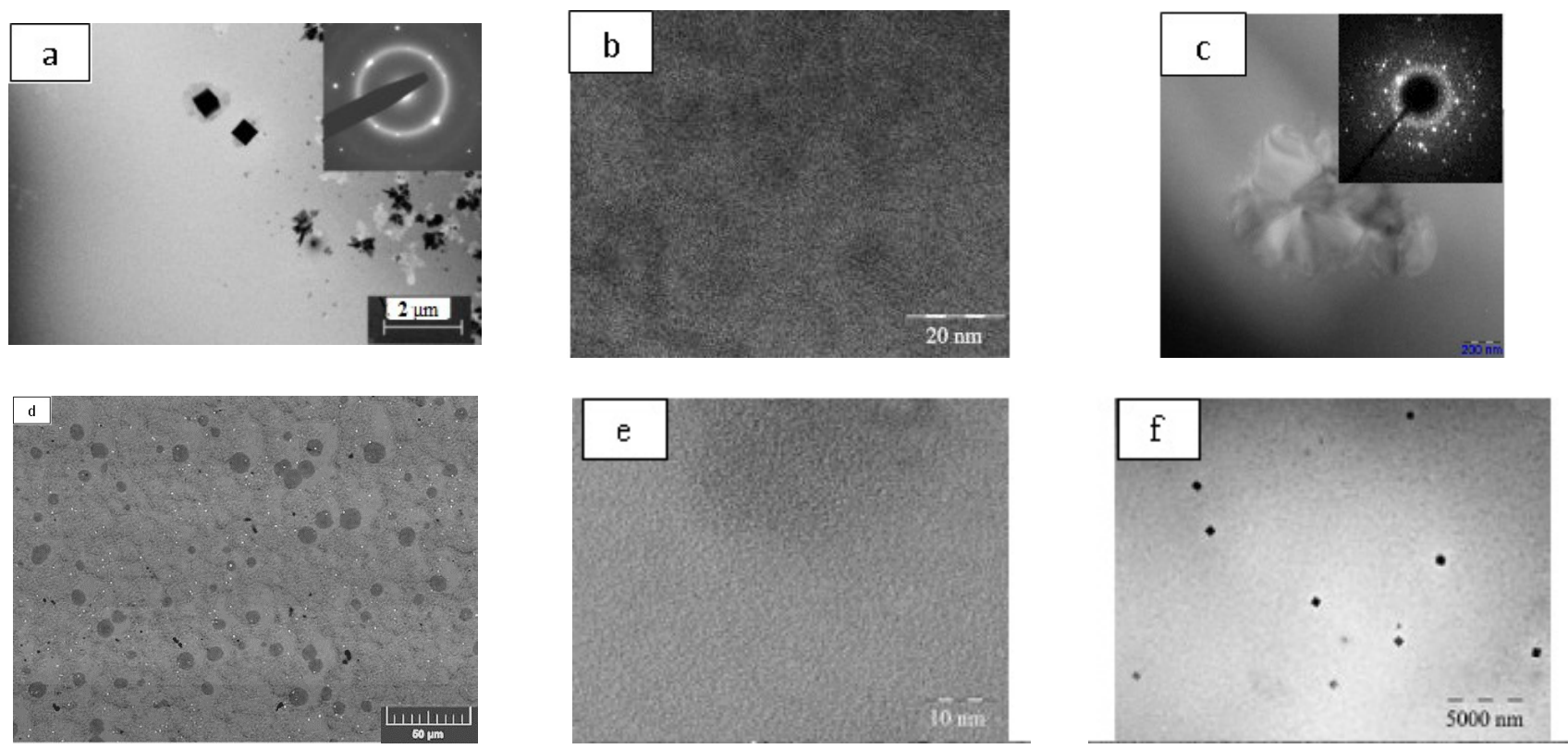

Рис. 2. Микроструктура $\mathrm{Zr}_{62} \mathrm{Cu}_{22} \mathrm{Al}_{10} \mathrm{Fe}_{5} \mathrm{Dy}$ ОМС: (a) исходное ОМС, светлое поле и микродифракция, СПЭМ; (b) исходное ОМС, светлое поле с большим увеличением; (c) СПЭМ - изображение кристаллической частицы - сферолита в литом ОМС сплаве; (d) ПЭМ-изображение конгломерата сферолитов - зерен и микродифракция от них, наблюдаемых в исходном ОМС; (е) ОМС после ИПДК при $20^{\circ} \mathrm{C}$, светлое поле; (f) ОМС после ИПДК при $150^{\circ} \mathrm{C}$, светлое поле.

Fig. 2. Microstructure of $\mathrm{Zr}_{62} \mathrm{Cu}_{22} \mathrm{Al}_{10} \mathrm{Fe}_{5} \mathrm{Dy}$ BMG: (a) the initial BMG, bright field and microdiffraction, STEM; (b) the initial BMG, bright field with a large magnification; (c) STEM image of the initial as-cast alloy showing crystalline particles - spherolites in the as-cast BMG alloy; (d) TEM image of a conglomerate of spherolites - grains, observed in the initial BMG, and microdiffraction taken from them; (e) $\mathrm{BMG}$ after $\mathrm{HPT}$ at $20^{\circ} \mathrm{C}$, bright field; (f) $\mathrm{BMG}$ after $\mathrm{HPT}$ at $150^{\circ} \mathrm{C}$, bright field. 
Табл. 1. Механические свойства на растяжение сплава $\mathrm{Zr}_{62} \mathrm{Cu}_{22} \mathrm{Al}_{10} \mathrm{Fe}_{5} \mathrm{Dy}_{1}$.

Table 1. Tensile mechanical properties of the $\mathrm{Zr}_{62} \mathrm{Cu}_{22} \mathrm{Al}_{10} \mathrm{Fe}_{5} \mathrm{Dy}_{1}$ alloy.

\begin{tabular}{|c|c|}
\hline $\begin{array}{c}\text { Состояние } \\
\text { State }\end{array}$ & $\begin{array}{c}\text { Максимальное напряжение } \\
\text { разрушения, МПа } \\
\text { Maximum fracture stress, MPa }\end{array}$ \\
\hline $\begin{array}{c}\text { Initial } \mathrm{BMG} \\
\mathrm{Zr}_{62} \mathrm{Cu}_{22} \mathrm{Al}_{10} \mathrm{Fe}_{5} \mathrm{Dy}_{1}\end{array}$ & 1410 \\
\hline $\mathrm{HPT}, T=20^{\circ} \mathrm{C}$ & 250 \\
\hline $\mathrm{HPT} n=5, T=150^{\circ} \mathrm{C}$ & 1240 \\
\hline
\end{tabular}
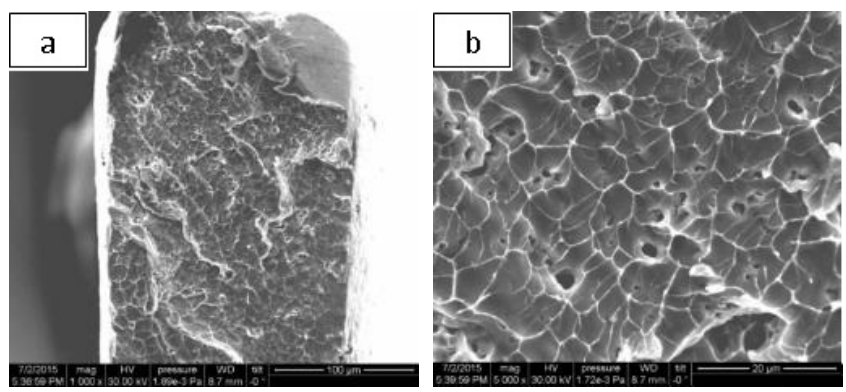

Рис. 3. Фрактография (РЭМ) излома после испытаний на растяжение исходного образца ОMC $\mathrm{Zr}_{62} \mathrm{Cu}_{22} \mathrm{Al}_{10} \mathrm{Fe}_{5} \mathrm{Dy}_{1}$ :(a) увеличение 1000; (b) увеличение 5000.

Fig. 3. Fractography (SEM) of the fracture sufrace after tensile testing of the initial $\mathrm{Zr}_{62} \mathrm{Cu}_{22} \mathrm{Al}_{10} \mathrm{Fe}_{5} \mathrm{Dy}_{1} \mathrm{BMG}$ sample: (a) at $1000 \mathrm{x}$ magnification; (b) at 5000x magnification.
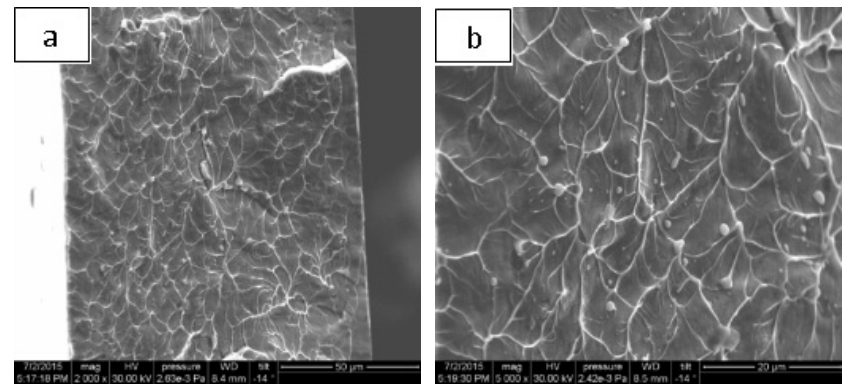

Рис. 4. Фрактография (РЭМ) излома после испытаний на растяжение образца ОMC $\mathrm{Zr}_{62} \mathrm{Cu}_{22} \mathrm{Al}_{10} \mathrm{Fe}_{5} \mathrm{Dy}_{1}$ ИПДК $n=5 T=$ $20^{\circ} \mathrm{C}$ :(a) увеличение 1000 ; (b) увеличение 5000.

Fig. 4. Fractography (SEM) of the fracture surface after tensile testing of the $\mathrm{Zr}_{62} \mathrm{Cu}_{22} \mathrm{Al}_{10} \mathrm{Fe}_{5} \mathrm{Dy}_{1} \mathrm{BMG}$ sample after HPT $n=5 \mathrm{~T}=20^{\circ} \mathrm{C}$ : (a) at $1000 x$ magnification; (b) at 5000x magnification.

\section{Заключение}

В соответствии с данными РСА и ПЭМ исходные стержни ОМС $\mathrm{Zr}_{62} \mathrm{Cu}_{22} \mathrm{Al}_{10} \mathrm{Fe}_{5} \mathrm{Dy}_{1}$ имеют аморфную структуру. После ИПДК при $20^{\circ} \mathrm{C}$ и $150^{\circ} \mathrm{C}$ структура ОМС $\mathrm{Zr}_{62} \mathrm{Cu}_{22} \mathrm{Al}_{10} \mathrm{Fe}_{5} \mathrm{Dy}_{1}$ остается аморфной. На данном этапе исследований методом РСА ПЭМ заметной разницы в структуре исходных ОМС и после ИПДК выявить не удалось.

Образцы аморфных сплавов $\mathrm{Zr}_{62} \mathrm{Cu}_{22} \mathrm{Al}_{10} \mathrm{Fe}_{5} \mathrm{Dy}_{1}$ до и после ИПДК при испытаниях на растяжение при различных температурах, включая $300^{\circ} \mathrm{C}$, разрушаются хрупко при напряжениях около 1500 МПа. Однако вид излома образцов ОМС до и после ИПДК после испытаний на растяжение несколько различается, что отражает изменение структуры аморфных сплавов в результате ИПДК.

Благодарность/Acknowledgements. Исследование выполнено за счет гранта Российского научного фонда (проект 14-12-00138). Механические испытания выполнены при поддержке гранта Президента Российской Федераичи для государственной поддержки ведущих научных школ № НШ-7996.2016.8. При исследованиях использовалось оборудование Ресурсных иентров СПБГУ, ЦКП «Нанотех» УГАТУ и отдела электронной микроскопии ЦКП «Испытательный иентр нанотехнологий и перспективных материалов» ИФМ УрО РАН.

\section{Литература/References}

1. Inoue, Stabilization of metallic supercooled liquid and bulk amorphous alloys Acta Mater. 48, 279 (2000).

2. A.L. Greer. Metallic glasses. Science. 267, 1947-1947 (1995).

3. V.Yu. Zadorozhnyy, A. Inoue, D. V. Louzguine-Luzgin. Mater. Sci. Eng. A. 551, 82 - 86 (2012).

4. R.Z. Valiev. Nature Materials. 3, $511-516$ (2004).

5. R.Z. Valiev, A.P. Zhilyaev, T.G. Langdon, Bulk Nanostructured Materials: Fundamentals and Applications, by John Wiley \& Sons, Inc., 456 (2014).

6. R.Z. Valiev, M.J. Zehetbauer, Yu. Estrin et al. Adv. Eng. Mater. 9 (7), 527 - 533 (2007).

7. R. Valiev, R. Islamgaliev, I. Alexandrov. Prog. Mat. Sci. 45, $103-189$ (2000).

8. A.A. Churakova, D.V. Gunderov, A.V. Lukyanov, Yu. A. Lebedev. Letters on materials. 3 (2),166-168 (2013). (in Russian) [А.А. Чуракова, Д. В. Гундеров, А. В. Лукьянов, Ю.А. Лебедев. Письма о материалах. 3, $166-168$ (2013)]

9. A.G. Popov, V.S. Gaviko, N.N. Shchegoleva, T.Z. Puzanova, A.S. Ermolenko, V.V. Stolyarov, D. V. Gunderov, G.I. Raab, R.Z. Valiev. The Physics of Metals and Metallography. 94 (1), (2002).

10. V. V. Stolyarov, D. V. Gunderov, R.Z. Valiev, A. G. Popov, V.S. Gaviko, A.S. Ermolenko. JMMM.196 - 197, 166 - 168 (1999).

11. A. V. Sergueeva, C. Song, R.Z. Valiev, A. K. Mukherjee. Mater. Sci. Eng. A.339 (1), 159 - 165 (2003).

12. D. Gunderov, A. Lukyanov, E. Prokofiev, A. Kilmametov, V. Pushin, R. Valiev. Materials Science and Engineering A. 503, 75 - 77 (2009).

13. D.V. Gunderov, A.V. Lukyanov, E.A. Prokofev, R.Z. Valiev, N.N. Kuranova, A.N. Uksusnikov, L. I. Yurchenko, V.G. Pushin. The Physics of Metals and Metallography. 108 (2), $131-138$ (2009). (in Russian) [Гундеров Д.В., Куранова Н.Н., Лукьянов А.В., Уксусников А.Н., Прокофьев Е.А., Юрченко Л.И., Валиев Р.3., Пушин В.Г. Физика металлов и металловедение.108 (2), 139 - 146 (2009)].

14. D. Gunderov, N. Kuranova, A. Lukyanov, V. Makarov, E. Prokofiev, A. Pushin. Rev. Adv. Mater.Sci. 25, 58-66 (2010) 
15. R.Z. Valiev, D. V. Gunderov, A. V. Lukyanov, V. G. Pushin. Journal of Materials Science. 47 (22), 7848 (2012).

16. N.N. Kuranova, V.V. Makarov, V.G. Pushin, A.N. Uksusnikov, R.Z. Valiev, D.V. Gunderov, A. V. Lukyanov, E. A. Prokofev. Bulletin of the Russian Academy of Sciences: Physics. 73 (8), 1117 - 1119 (2009).

17. A.M. Glezer, M.P. Plotnikova, A.V. Shalimova, S. V. Dobatkin. Bulletin of the Russian Academy of Sciences: Physics. 73 (9), 1233 - 1239 (2009).

18. R.Z. Valiev, D. V. Gunderov, V.G. Pushin, A. G. Popov. Doklady Physics. 49 (9), 519-521 (2004). (in Russian) [Р.3. Валиев, В.Г. Пушин, Д. В. Гундеров, А. Г. Попов. Докл. РАН. 398 (1), 54 (2004)].

19. Li Hailing, Li Lou, Fuchen Hou, Defeng Guo, Wei Li, Xiaohong Li, D. V. Gunderov, Kiminori Sato, Xiangyi Zhang. 103, 142406 (2013)

20. D. V. Gunderov, V.Yu. Slesarenko, A.V. Lukyanov, A.A. Churakova, E.V. Boltynjuk, V.G. Pushin, E. V. Ubyivovk, A. V. Shelyakov, R.Z. Valiev. Advanced Engineering Materials. 17 (12), 1728 - 1732 (2015).
21. D. V. Gunderov, V.Yu. Slesarenko, A.A. Churakova, A. V. Lukyanov, E. P. Soshnikova, V. G. Pushin, R. Z. Valiev. Intermetallics. 66, $77-81$ (2015). doi:10.1016/j. intermet.2015.06.013

22. E. I. Teitel, L. S. Metlov, D. V. Gunderov, A. V. Korznikov. The Physics of Metals and Metallography. 113 (12), 1162-1168 (2012). (in Russian) [И. Тейтель, Л.С. Метлов, Д.В. Гундеров, А.В. Физика металлов и металловедение. 113 (12), 1230 (2012)].

23. V.Yu. Slesarenko, D. V. Gunderov, P.G. Ulyanov, R.Z. Valiev. IOP Conf. Series: Materials Science and Engineering. 63, 012166 (2014). doi:10.1088/1757899X/63/1/012166

24. E.V. Boltynjuk, D. V. Gunderov, E.V. Ubyivovk, A. V. Lukianov, A. M. Kshumanev, A. Bednarz, R. Z. Valiev. STRANN AIP Conf. Proc. 1748, 030006-1 - 030006-6 (2016). doi: 10.1063/1.4954352

25. A. Yu. Churyumov, A.I. Bazlov, A.A. Tsarkov, A. N. Solonin, D. V. Louzguine-Luzgin, J. Alloys Comp. 654, $87-94$ (2016). 